\title{
The role of brain metastases in patients with pulmonary adenocarcinoma: a retrospective study
}

\author{
Jingyuan Xue ${ }^{1,2 \#}$, Meirong Liu ${ }^{1,3 \#}$, Hui Zhao ${ }^{1,3}$, Xiaoqing Li $^{1}$, Qiong Qin ${ }^{1}$, Yaoyao Ren ${ }^{1}$, Diansheng Zhong ${ }^{1}$ \\ ${ }^{1}$ Department of Oncology, Tianjin Medical University General Hospital, Tianjin, China; ${ }^{2}$ Department of Geriatrics, Tianjin Medical University \\ General Hospital, Tianjin, China; ${ }^{3}$ Department of Infectious Diseases, Tianjin Hospital, Tianjin, China \\ Contributions: (I) Conception and design: D Zhong; (II) Administrative support: D Zhong; (III) Provision of study materials or patients: All authors; (IV) \\ Collection and assembly of data: J Xue, M Liu; (V) Data analysis and interpretation: J Xue, M Liu; (VI) Manuscript writing: All authors; (VII) Final \\ approval of manuscript: All authors. \\ \#These authors contributed equally to this work. \\ Correspondence to: Diansheng Zhong. Department of Oncology, Tianjin Medical University General Hospital, 154 Anshan Street, Heping District, \\ Tianjin 300052, China. Email: oncologyzhong@163.com.
}

\begin{abstract}
Background: Brain metastasis is common in patients with lung cancer and has a negative impact on overall survival (OS). The aim of this study was to investigate therapeutic effects and prognosis-related factors for survival of patients with pulmonary adenocarcinoma and brain metastasis.

Methods: This was a retrospective study involving the data of patients with pulmonary adenocarcinoma and brain metastasis who were treated at the Department of Oncology, Tianjin Medical University General Hospital. Participants were divided into 2 groups: the initial-metastasis group (brain metastases at first visit), and the late-metastasis group (developed metastasis during treatment). The quantitative index was described as mean \pm standard deviation (SD), independent sample t-test was used to calculate the $\mathrm{P}$ value. All data were processed using the software SPSS 20.0 software package.
\end{abstract}

Results: There were a total of 43 patients in the initial-metastasis group and 49 in the late-metastasis group. First-line treatment efficacy $(\mathrm{P}=0.004)$, $\mathrm{OS}(\mathrm{P}<0.001)$, and progression-free survival $(\mathrm{PFS})(\mathrm{P}=0.006)$ were higher in the late-metastasis group. Kaplan-Meier curves revealed that the late-metastasis group had longer OS, intracranial progression-free survival (IPFS), and PFS (all $\mathrm{P}<0.001$ ). Multivariate analysis showed that gender, Karnofsky performance status (KPS), efficacy of first-line treatment, radiotherapy, and brain metastasis at initial visit were significantly related to OS.

Conclusions: Participants with pulmonary adenocarcinoma and brain metastasis at their initial visit had shorter OS and PFS than those who developed brain metastasis later. The efficacy of first-line treatment was related to OS while female gender and high KPS score were protective factors.

Keywords: Carcinoma; non-small cell lung cancer (NSCLC); neoplasm metastasis; survival analysis

Submitted Sep 28, 2021. Accepted for publication Nov 29, 2021.

doi: 10.21037/apm-21-3146

View this article at: https://dx.doi.org/10.21037/apm-21-3146

\section{Introduction}

Lung cancer is the most common cause of cancer death worldwide and accounts for $13 \%$ of total cancer diagnoses (1). Approximately $85 \%$ of the pathological types of lung cancer are non-small cell lung cancer (NSCLC), the most common type of NSCLC is pulmonary adenocarcinoma. The prognosis of patients with NSCLC is poor due to most patients already being in late stage of cancer at the time of diagnosis (2). Distant metastasis is often observed at presentation; $10-20 \%$ of patients with NSCLC will present with brain metastasis at first diagnosis (3) and up to $50 \%$ will develop brain metastasis over the course of the disease (4). The self-reported health-related quality 
of life of patients who are diagnosed with brain metastasis at their initial diagnosis deteriorates faster than those who present without brain metastasis (5). Brain metastases are more frequent in NSCLCs with oncogenic driver mutations like epidermal growth factor receptor (EGFR)-mutation or anaplastic lymphoma kinase (ALK)-rearrangement, ranging between $25 \%$ at diagnosis to more than $45 \% 3$ years after diagnosis (6). To our knowledge, survival in NSCLC with brain metastases was poor (7) which has improved with recent advancements.

The choice of therapeutic approach for brain metastasis should be guided by knowledge of a number of prognostic factors, including the patient's age, primary/systemic tumor activity, neurocognitive function, Karnofsky performance status (KPS), number of metastases, and time from first diagnosis (8). There is some concern over the effectiveness of drug treatment because of the blood-brain barrier (BBB), but many agents are effective as the BBB has already been breached by the metastatic lesion (9).

Numerous pulmonary adenocarcinomas exhibit single oncogene aberrations that play major roles in oncogenesis and tumor progression, thus treatment has been improved recently with adoption of targeted therapy (10). For example, EGFR mutations exist in many Asian populations with pulmonary adenocarcinoma and respond well to therapy with tyrosine kinase inhibitors (TKIs) that suppress the aberrant EGFR kinase activity (11). However, patients with $E G F R$ mutation have a higher rate of brain metastasis than those with EGFR-wild-type NSCLC (12) and they are more likely to develop multiple metastases (13). Immunotherapy and chemotherapy are also important approaches, particularly for patients who are EGFR-wildtype (14).

Overall, the survival of patients with NSCLC and brain metastasis remains unsatisfactory (15). The treatment options are quite complex, and many factors influence the outcomes of patients with NSCLC (16). Thus, more studies are needed to fully elucidate the optimal approach for treating patients with NSCLC that has metastasized to the brain (17). However, there were few studies on the different time of brain metastasis in patients with stage IV pulmonary adenocarcinoma. The purpose of our study was to investigate the survival of patients with pulmonary adenocarcinoma and brain metastasis and examine therapeutic effects and prognosis-related factors in these patients. We present the following article in accordance with the STROBE reporting checklist (available at https:// dx.doi.org/10.21037/apm-21-3146).

\section{Methods}

\section{Study design}

This was a retrospective study of clinical data from patients with pulmonary adenocarcinoma who developed brain metastasis and were treated at the Department of Oncology, Tianjin Medical University General Hospital between 1 January 2012 and 31 September 2018. The study was conducted in accordance with the Declaration of Helsinki (as revised in 2013). The study was approved by the Ethics Committee of Tianjin Medical University General Hospital (approval No. 201171). Consents were provided by the participants.

\section{Patients}

The inclusion criteria for this study were as follows: (I) stage IV pulmonary adenocarcinoma with brain metastases at final diagnosis that was confirmed pathologically and by imaging [staging was performed in accordance with the $8^{\text {th }}$ edition of American Joint Committee on Cancer Staging (AJCC) Cancer Staging Manual, for NSCLC (18)]; (II) brain metastases that were untreated or had received operation, targeted therapy, chemotherapy, or radiotherapy; (III) KPS of 60-100 at the initial visit; (IV) the patient's organ function met the following criteria at the initial visit: bone marrow function: neutrophil (NEUT) \# $\geq 1.5 \times 10^{9} / \mathrm{L}$, hemoglobin $(\mathrm{HGB}) \geq 60 \mathrm{~g} / \mathrm{L}$, platelet (PLT) $\# \geq 80 \times 10^{9} / \mathrm{L}$; liver function: bilirubin $\leq$ upper limit of normal (ULN), alanine transaminase (ALT) and aspartate transaminase (19) $\leq \mathrm{ULN}$; renal function: creatinine clearance rate $(\mathrm{CCR}) \geq 30 \mathrm{~mL} / \mathrm{h}$; coagulation function: bleeding and clotting time $\leq \mathrm{ULN}$.

The exclusion criteria were as follows: (I) no pathological evidence or pathologically diagnosed non-pulmonary adenocarcinoma or pulmonary adenocarcinoma without brain metastases by the final imaging diagnosis; (II) unclear clinical staging, incomplete data, and loss to follow-up; (III) patients with a primary intracranial tumor or those with non-pulmonary adenocarcinoma complicated by brain metastases; (IV) patients with systemic diseases needing long-term medication with glucocorticoid drugs; (V) severe clinical infection or organ failure, or hemodynamic instability needing admission to the intensive care unit (ICU).

The eligible patients were divided into 2 groups, an initial-metastasis group and alate-metastasis group, on the basis of whether the pulmonary adenocarcinoma was accompanied by brain metastases at the initial visit or was diagnosed at a later stage. 


\section{Treatments}

All participants received one or multiple therapies according to the recommendation of the treating clinician and with their consent. These included surgical procedures to resect the intrapulmonary and/or intracranial tumor, chemotherapy, radiotherapy of the intrapulmonary and/or intracranial tumor, targeted therapy, bio-immunotherapy, or no treatment (best supportive care).

\section{Data collection}

Participants' data were collected from their medical records regarding age, gender, whether there was metastasis at the initial visit, whether there were symptoms confirming metastasis, location of metastases, therapeutic regimens, first-line therapeutic effect, overall survival (OS), intracranial progression-free survival (IPFS), and progression-free survival (PFS).

We defined OS as duration from first-line treatment to death resulting from any cause (the time of the final followup visit for those who were lost to follow up; the day of the end of follow-up visit for those still alive at the end of the study).

We defined PFS as time horizon from first-line treatment to first tumor progression or death.

The therapeutic effect was defined according to complete response (CR), partial response (PR), stable disease (SD), and progressive disease (PD) (20).

The identification of metastasis was confirmed by histopathological results and diagnosis of an intracranial metastatic tumor by double-dose enhanced magnetic resonance imaging (MRI) or enhanced computed tomography (CT) scan of the head, and by correlating these images with clinical symptoms and signs or after intracranial operation brain metastases were pathologically confirmed to be derived from the pulmonary adenocarcinoma.

\section{Statistical analysis}

Quantitative indexes that were normally distributed were described by mean \pm standard deviation $(\mathrm{SD})$, and independent-samples $t$-test was used to calculate $\mathrm{P}$ values; those that were not normally distributed were described by median (range), and a non-parametric test was used to calculate $\mathrm{P}$ values. Classification indexes were described by using frequency (percentage), and chi-square test or Fisher exact test (if the expected frequency of over $20 \%$ of cells was less than 5) were used to compare independent samples in the 2 groups. Taking OS, PFS, and IPFS as outcomes, the grouping was performed based on whether there were brain metastases at the initial visit, and a Kaplan-Meier curve was drawn, and log-rank test was used to determine whether survival curves were significantly different. The OS, PFS, and IPFS were regarded as outcomes for Cox regression analysis, and factors with significant results of univariate analysis $(\mathrm{P}<0.05)$ were included in the multivariate analysis. All statistical tests were performed with the SPSS 20.0 software package (IBM Corp., Armonk, NY, USA) and the use of two-sided test, and $\mathrm{P}$ values of less than 0.05 were considered to indicate statistical significance.

\section{Results}

\section{Baseline characteristics}

The baseline data from the 2 participant groups are shown in Table 1. There were 43 patients diagnosed with metastasis at the initial visit who were allocated to the initial-metastasis group and 49 patients who showed no metastasis at the initial visit who were allocated to the late-metastasis group. The two groups were similar in terms of gender, age, and smoking pack year. There was a significantly higher KPS score in the late-metastasis group $(80.21 \pm 3.85$ vs. $76.50 \pm 7.00, \mathrm{P}=0.002)$. The primary site of the adenocarcinoma, tumor size, staging, and genetic testing showed no difference between the groups. More participants in the late-metastasis group underwent surgery than in the initial-metastasis group ( $34.7 \%$ vs. $11.6 \%, \mathrm{P}=0.019)$ but the other first-line treatment methods were similar between the groups. It could be seen the $25 \%$ of participants in the latemetastasis group had brain metastases within 182 days, and the other $75 \%$ of participants had brain metastases within 556 days. The average time of brain metastasis was 399 days in the late-metastasis group.

\section{First-line treatment outcomes}

The efficacy of the first-line treatment is also shown in Table 1. The late-metastasis group had a median OS of 617 days compared with 279 days of the initial-metastasis group $(\mathrm{P}<0.001)$. PFS was significantly higher in the latemetastasis group than that in initial-metastasis group (259 vs. 161 days, $\mathrm{P}=0.006)$. 
Table 1 Baseline data of the two patient groups

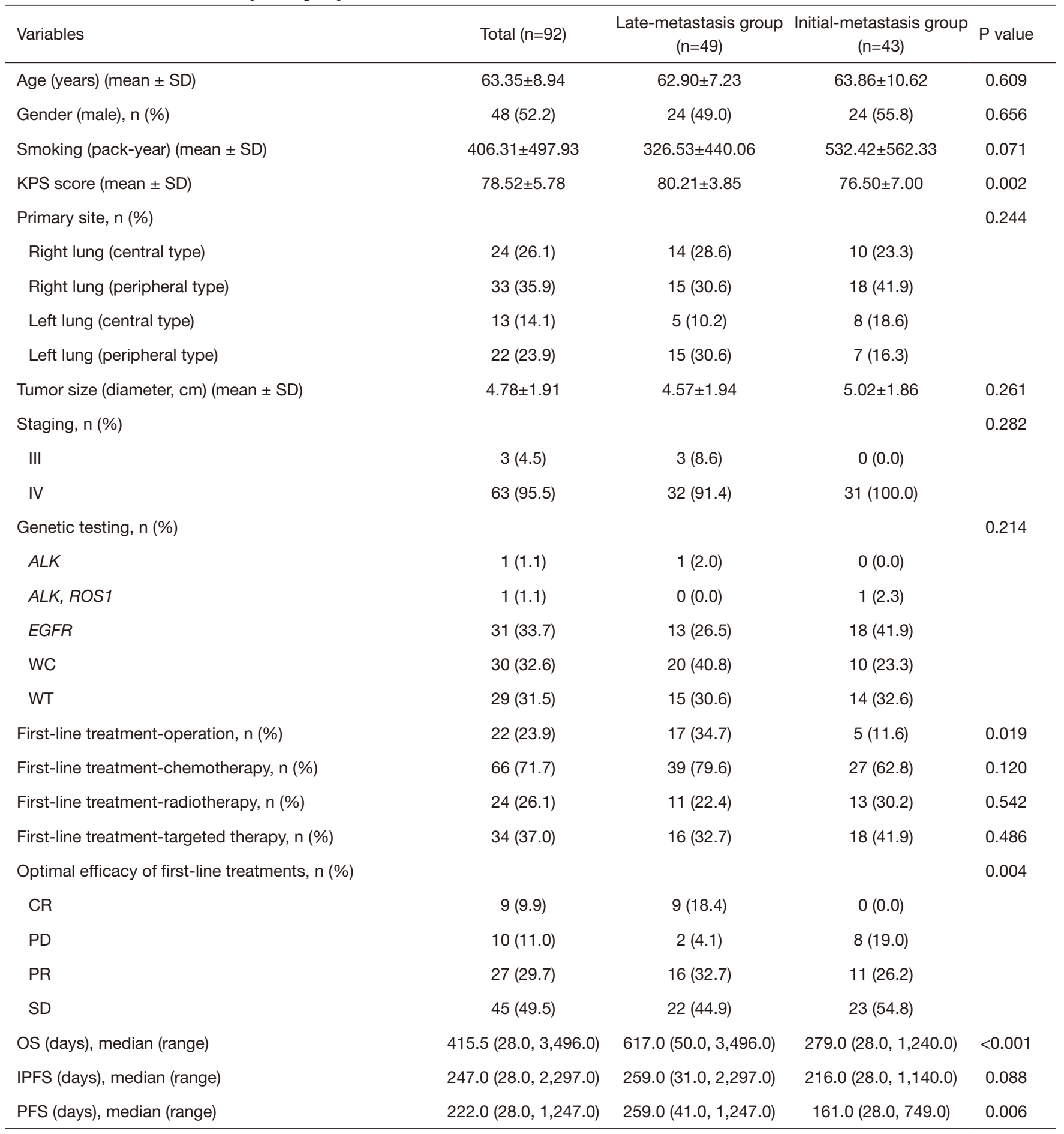

KPS, Karnofsky performance score; ALK, anaplastic lymphoma kinase; EGFR, epidermal growth factor receptor; WC, undetected; WT, wild type; CR, complete response; PD, progressive disease; PR, partial response; SD, stable disease; OS, overall survival; IPFS, intracranial progression-free survival; PFS, progression-free survival. 
Table 2 Clinical data related to brain metastases

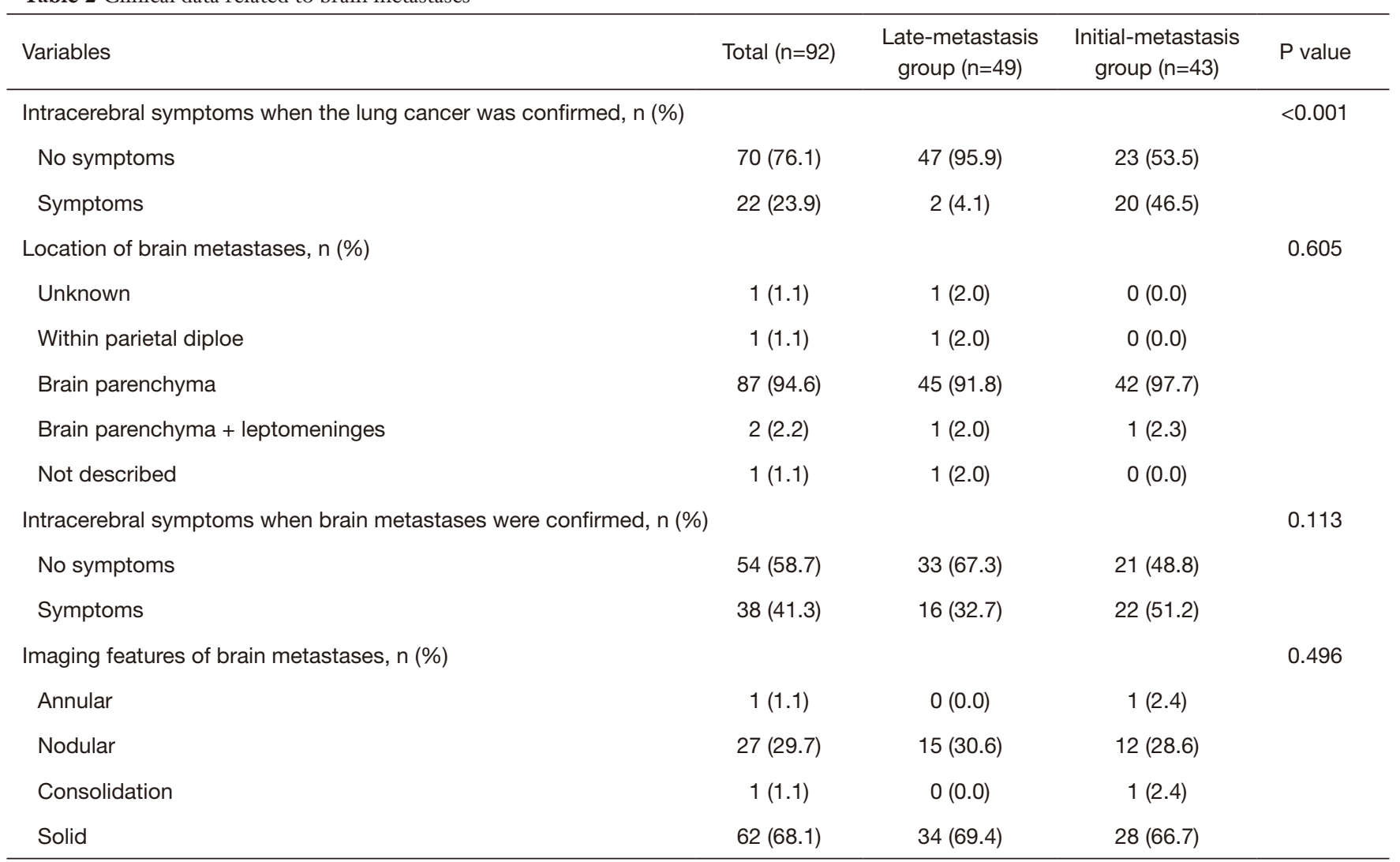

\section{Brain metastasis results}

The clinical data related to the development of brain metastases in these participants is described in Table 2. Initial-metastasis group participants showed more intracerebral symptoms when the lung cancer diagnosis was confirmed than those in the late-metastasis group (46.4\% vs. $4.1 \%, \mathrm{P}<0.001)$, but there was no difference in symptoms between the groups at the time of metastasis diagnosis. The location and imaging characteristics of the metastases were similar between the 2 groups.

\section{Survival analysis}

Kaplan-Meier curves showed that the participants in the late-metastasis group had longer OS, IPFS, and PFS than those in the initial-metastasis group (all $\mathrm{P}<0.001$, Figure 1).

\section{Cox regression analysis of $O S, P F S$, and IPFS}

The analysis of factors related to OS in the patients with pulmonary adenocarcinoma is shown in Figure 2. Univariate analysis showed that gender, KPS, tumor size, optimal efficacy of first-line treatment, involvement of surgery in the first-line treatment, involvement of radiotherapy in the first-line treatment, and brain metastasis at initial visit were all significant for OS. When analyzed by multivariate analysis, gender, KPS, optimal efficacy of firstline treatment, involvement of radiotherapy in the firstline treatment, and brain metastasis at initial visit remained significant factors related to OS.

The analysis of factors related to PFS is shown in Figure 3. Univariate analysis suggested that KPS, optimal efficacy of first-line treatment, involvement of surgery in the first-line treatment, and brain metastasis at initial visit were all significant for PFS. When analyzed by multivariate analysis, optimal efficacy of first-line treatment (but not $\mathrm{CR}$ vs. PD) and brain metastasis at initial visit remained significant factors related to PFS.

The analysis of factors related to IPFS is illustrated in Figure 4. Univariate analysis showed that smoking pack year, KPS, optimal efficacy of first-line treatment, involvement of surgery in the first-line treatment, and brain metastasis 

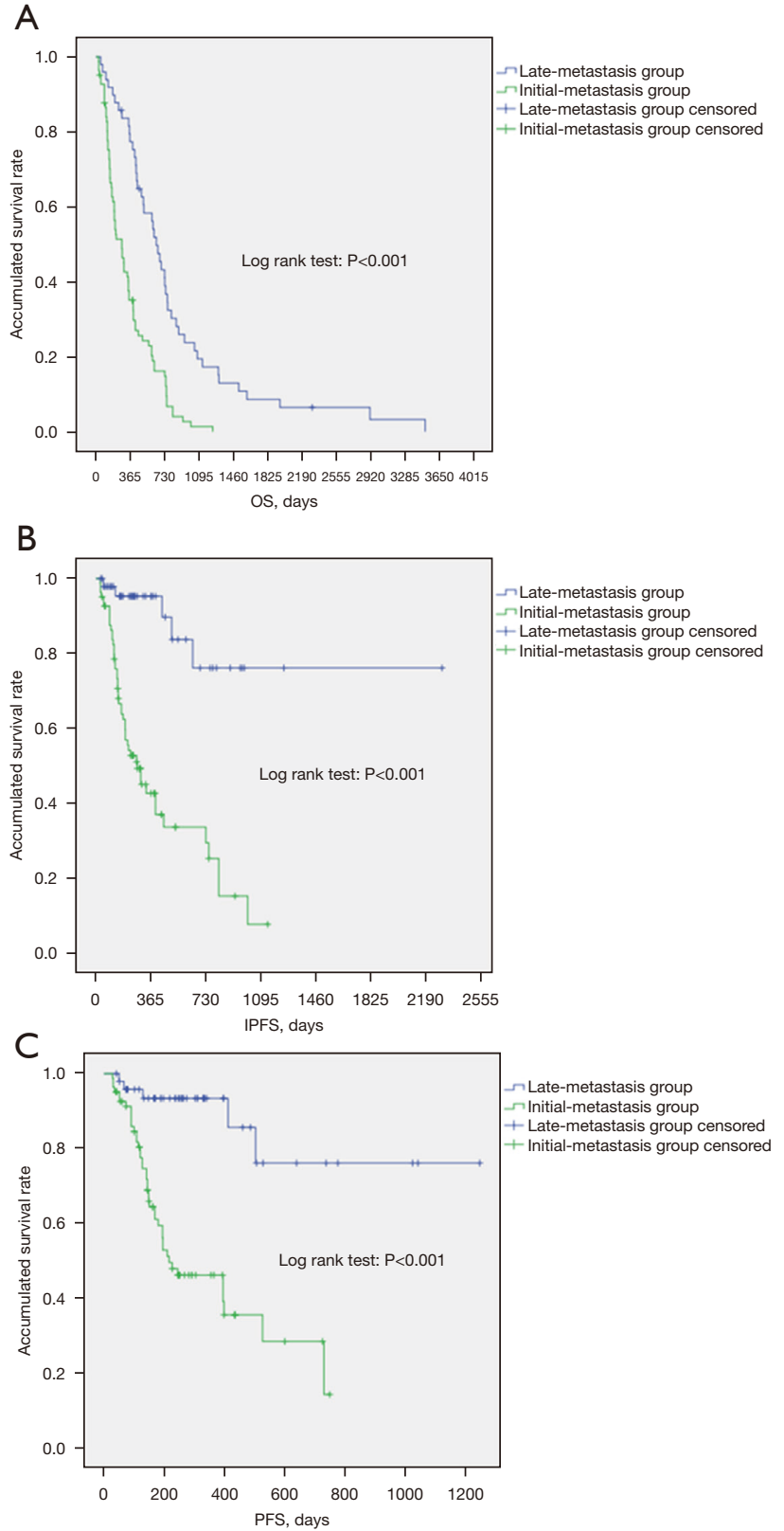

Figure 1 Survival analysis of the two patient groups using KaplanMeier curves. The late-metastasis group is shown by the blue line. The initial-metastasis group is shown by the green line. (A) OS of the late-metastasis group was significantly longer than the initial-metastasis group. (B) IPFS of the late-metastasis group was significantly longer than the initial-metastasis group. (C) PFS of the late-metastasis group was significantly longer than the initial-metastasis group. OS, overall survival; IPFS, intracranial progression-free survival; PFS, progression free survival. at initial visit were all significant for IPFS. Multivariate analysis showed that optimal efficacy of first-line treatment and brain metastasis at initial visit remained significant factors related to IPFS.

\section{Discussion}

This study focuses on the correlation between brain metastasis and OS in patients with advanced lung cancer, and the correlation between brain metastasis and various clinical factors.

Smoking pack year was higher in the initial-metastasis group, KPS was higher in the late-metastasis group, and more patients in the late-metastasis group underwent surgery as first-line treatment. First-line treatment efficacy, OS, and PFS were higher in the late-metastasis group. Survival analysis with Kaplan-Meier curves showed that the late-metastasis group had longer OS, IPFS, and PFS. Multivariate analysis showed that gender, KPS, efficacy of first-line treatment, radiotherapy, and brain metastasis at initial visit were significantly related to OS and that efficacy of first-line treatment and brain metastasis at initial visit were both factors related to IPFS and PFS.

The results of this study revealed a clear relationship between the diagnosis of brain metastasis at the patient's first hospital appointment and shorter survival in terms of OS, PFS, and IPFS. The median OS of patients in the initial-metastasis group was 279 days (equal to 9.2 months), while in the late-metastasis group it was 617 days (equal to 20.3 months). These rates compare quite favorably to previous studies that showed a 6-month median survival of patients with brain metastasis at presentation (3), OS of patients with NSCLC and brain metastasis of 7.7 months for patients treated with chemotherapy plus bevacizumab or chemotherapy and targeted therapy (14), and median survival time of 8 months in NSCLC patients with brain metastases after radiosurgery (21). However, it remains evident that patients with brain metastasis have a poor outcome and that early diagnosis prior to metastasis is important for improving survival rates (2).

The efficacy of the first-line treatment regimen was shown to be important for longer OS, PFS, and IPFS in this study. A variety of regimens are used as first-line treatment in patients with advanced NSCLC. These regimens are selected based on a patient's histology, molecular profile, and performance 


\begin{tabular}{|c|c|c|c|c|c|c|c|}
\hline & ontrol grou & & $\mathrm{HR}(95 \% \mathrm{CI})$ & $P$ value & & Adjusted HR $(95 \% \mathrm{Cl})$ & $P$ value \\
\hline Gender & Female & $1-1$ & $1.656(1.152,2.382)$ & 0.006 & $1 \mapsto$ & $1.897(1.276,2.823)$ & 0.002 \\
\hline Age & & $\phi$ & $1.004(0.982,1.027)$ & 0.705 & & & \\
\hline Smoking pack-year & & $\phi$ & $1.000(1.000,1.001)$ & 0.078 & & & \\
\hline KPS & & 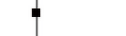 & $0.965(0.934,0.997)$ & 0.035 & $\phi$ & $0.964(0.931,0.998)$ & 0.040 \\
\hline Tumor size & & 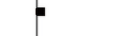 & $1.119(1.022,1.225)$ & 0.015 & $\phi$ & $1.037(0.931,1.154)$ & 0.512 \\
\hline Optimal efficacy & & & & $<0.001$ & & & $<0.001$ \\
\hline $\mathrm{SD}$ & $\mathrm{PD}$ & $=$ & $0.337(0.202,0.561)$ & $<0.001$ & - & $0.344(0.196,0.601)$ & $<0.001$ \\
\hline PR & $\mathrm{PD}$ & - & $0.183(0.101,0.331)$ & $<0.001$ & - & $0.147(0.074,0.290)$ & $<0.001$ \\
\hline $\mathrm{CR}$ & $\mathrm{PD}$ & - & $0.103(0.043,0.243)$ & $<0.001$ & - & $0.109(0.033,0.365)$ & $<0.001$ \\
\hline First-line operation & No & - & $0.482(0.302,0.770)$ & 0.002 & -1 & $0.833(0.430,1.615)$ & 0.589 \\
\hline First-line chemotherapy & No & & $0.803(0.541,1.193)$ & 0.803 & & & \\
\hline First-line radiotherapy & No & - & $0.553(0.368,0.832)$ & 0.004 & $\bullet$ & $0.323(0.194,0.538)$ & $<0.001$ \\
\hline First-line targeted therapy & No & & $0.863(0.594,1.253)$ & 0.438 & & & \\
\hline Brain metastases & No & $\longmapsto$ & $2.764(1.849,4.132)$ & $<0.001$ & $\longmapsto$ & $2.611(1.690,4.036)$ & $<0.001$ \\
\hline
\end{tabular}

Figure 2 Cox regression analysis taking OS as the outcome. OS, overall survival; HR, hazard ratio; CI, confidence interval; KPS, Karnofsky performance score; SD, stable disease; PR, partial response; CR, complete response.

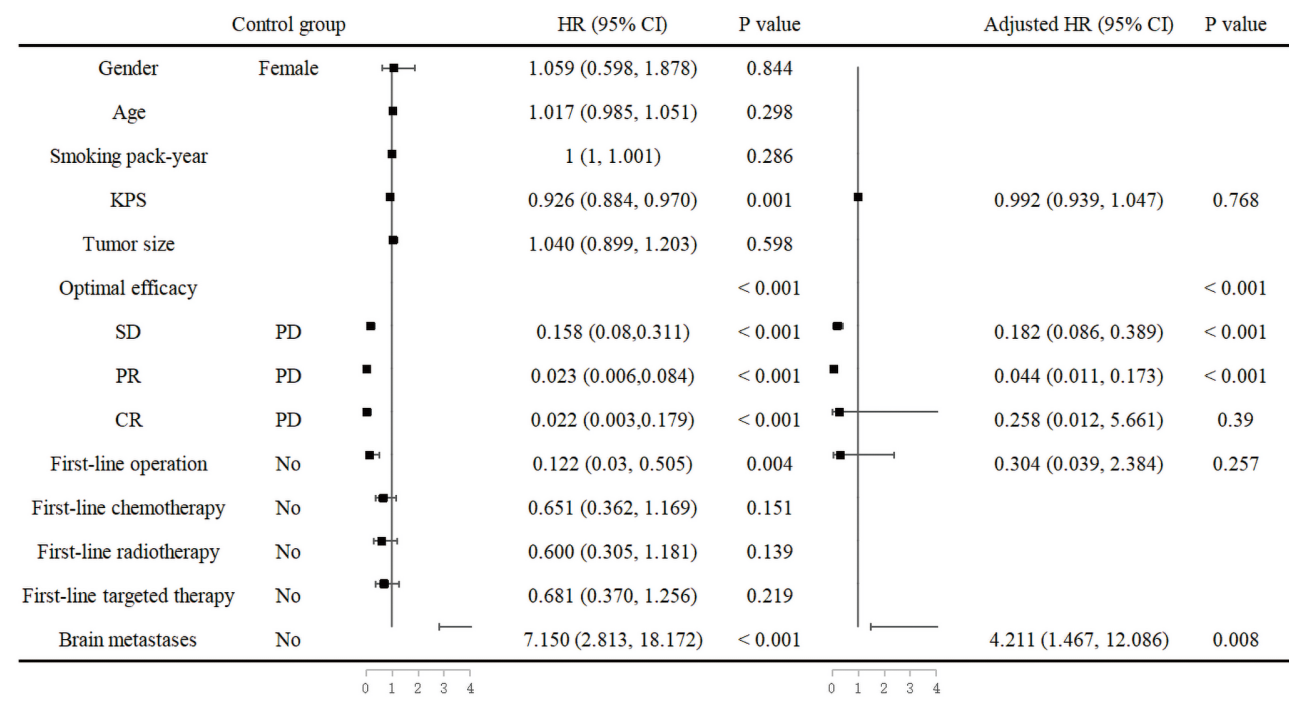

Figure 3 Cox regression analysis taking PFS as the outcome. PFS, progression free survival; HR, hazard ratio; CI, confidence interval; KPS, Karnofsky performance score; SD, stable disease; PD, progressive disease; PR, partial response; CR, complete response.

status, and include combination and single treatments (22). However, the optimal choice of first-line therapy for NSCLC is not always clear and more information is needed to assist clinicians with selection of the best regimen (23). Standard regimens for patients with stage IV NSCLC typically include chemotherapy involving targeted therapy and immunotherapy and palliative radiation; however, therapeutic surgery is also performed, and thoracic surgery can be beneficial for select patients (24). In this study, the use of radiotherapy showed a correlation to longer OS, which has been similarly reflected in the results of other studies $(25,26)$. However, another study in EGFR mutant patients found no long-term survival benefit with the use of radiotherapy (27). There also remains some debate about whether the benefits of radiotherapy outweigh the adverse events and its use should be carefully considered $(17,28)$. 


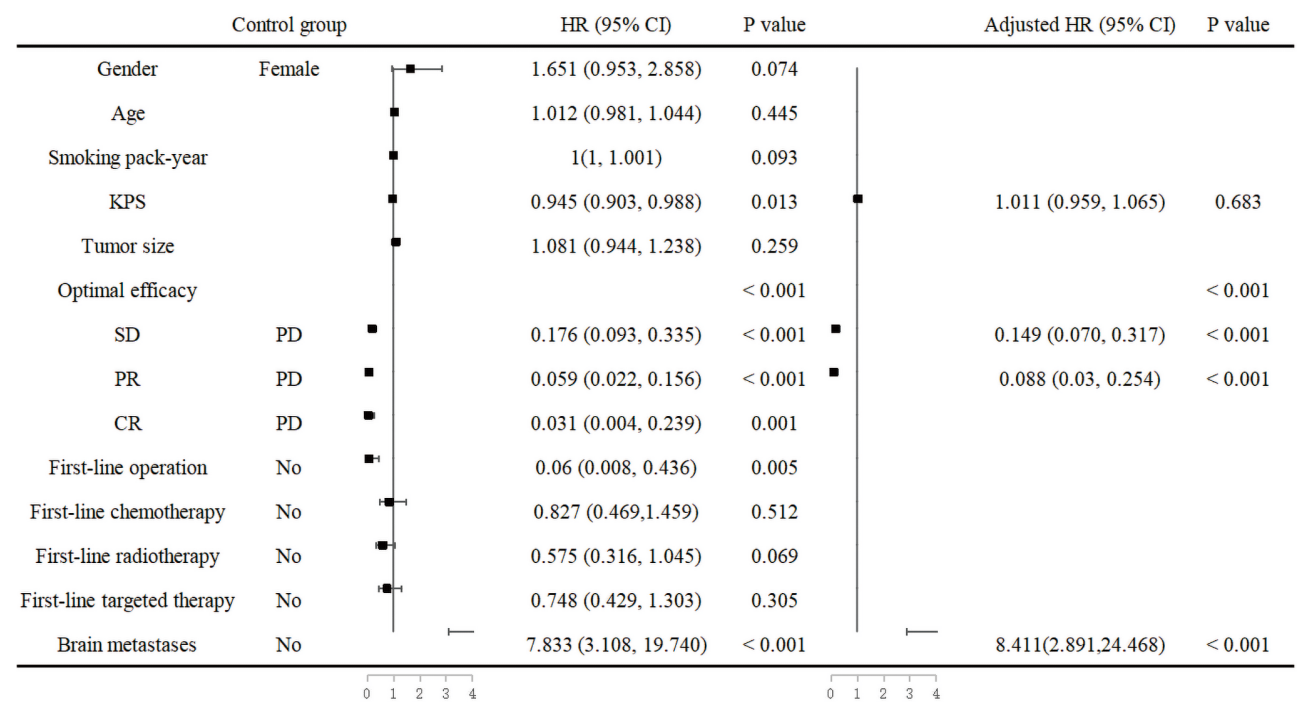

Figure 4 Cox regression analysis taking IPFS as the outcome. IPFS, intracranial progression-free survival; HR, hazard ratio; CI, confidence interval; KPS, Karnofsky performance score; SD, stable disease; PD, progressive disease; PR, partial response; CR, complete response.

Gender was also an important factor related to OS in this study although it showed no correlation to PFS. A study in patients with early-stage NSCLC treated with surgical resection found no gender difference in OS (29). However, female gender as well as pathological stage were favorable prognostic factors for survival after surgery in another study (30). Female gender also contributed to improved survival regardless of treatment following lobectomy for NSCLC larger than $4 \mathrm{~cm}$ (31).

The KPS value at diagnosis is a direct measure of a patient's functional status. This study showed that KPS was related to OS in this patient population, and this is supported by other studies. A study that aimed to predict the survival of patients with NSCLC and brain metastasis showed that KPS at diagnosis of brain metastasis was an independent predictor of survival (32). Another study showed that a KPS of $90 \%$ vs. $70-80 \%$ was a significant prognostic factor after radical treatment for oligometastatic NSCLC (33). The KPS was also an important prognostic factor for patients with NSCLC and brain metastasis after radiosurgery (21). At the other end of the scale, KPS is also related to prognosis in early-stage NSCLC (34). Therefore, KPS can be correctly used as an aid to prognosis assessment and selection of appropriate treatment regimen (33).

Although in this study we found no significant difference in the genetic testing results between the late-metastasis group and initial-metastasis group, previous studies have shown that patients with EGFR mutation have higher rates of brain metastasis than those without the mutation $(12,35)$. Asian populations with NSCLC tend to have a high prevalence of EGFR mutations (36). In this study, 33.7\% of participants were identified as having EGFR mutation. This is similar to the overall prevalence worldwide but slightly lower than a previous rate measured in China of $38.4 \%$ (37). This rate stood at $41.9 \%$ in the initial-metastasis group compared to $26.5 \%$ in the late-metastasis group, so a larger study may reveal a statistical difference for early metastasis in patients with EGFR mutation that was not evident in this study. According to this study, chemotherapy combined with TKI is the best choice for patients with stage IV lung cancer brain metastasis complicated with EGFR mutation (Figure 5).

Previous studies have shown that patients with intracranial symptoms are more likely to receive surgical treatment (4) or combination treatment with TKIs and radiotherapy rather than TKI treatment alone (38). So, differences in first-line treatment may influence the survival of patients. However, the time from the confirmation of lung cancer to the confirmation of brain metastases was not available and the appropriate multivariate analysis could not be performed. These aspects will require further investigation in future.

This study had some other limitations. As a retrospective analysis, it is likely that there was some selection bias in patient inclusion. This study was conducted in a single hospital department, so the sample size was relatively 


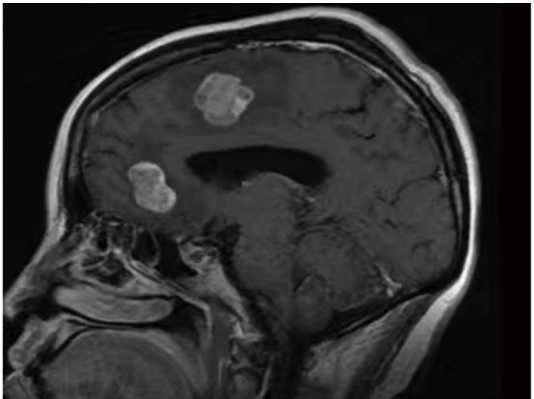

Before treatment

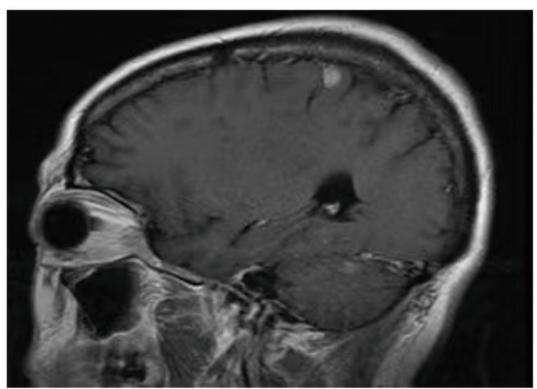

Before treatment

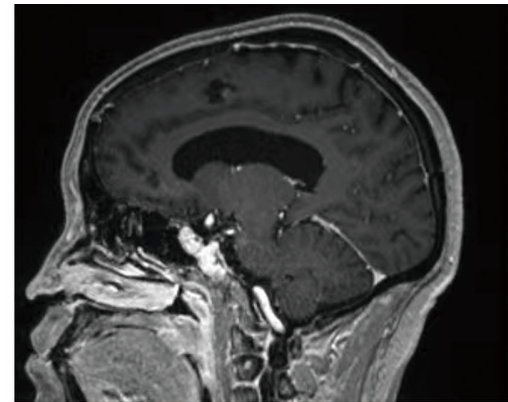

After treatment

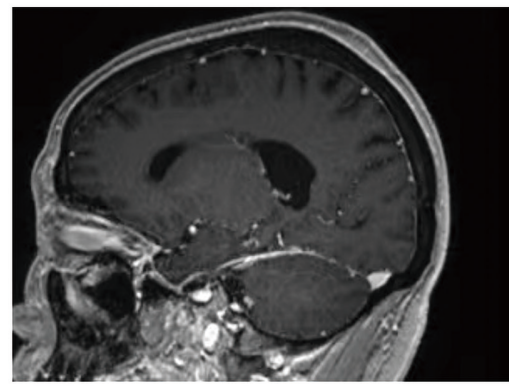

After treatment

Figure 5 Magnetic resonance imaging of the group with early-onset brain metastasis by chemotherapy combined with epidermal growth factor receptor tyrosine kinase inhibitor.

small. Larger scale studies including patients from multiple centers are needed to support our results.

The OS and PFS for patients with pulmonary adenocarcinoma and brain metastases present at their initial visit were shorter than those for patients who first presented without brain metastases. The better the first-line therapeutic effect was, the longer the OS, PFS, and IPFS. In addition, female gender and high KPS score were related factors for longer OS.

\section{Acknowledgments}

Funding: None.

\section{Footnote}

Reporting Checklist: The authors have completed the STROBE reporting checklist. Available at https://dx.doi. org/10.21037/apm-21-3146

Data Sharing Statement: Available at https://dx.doi. org/10.21037/apm-21-3146
Conflicts of Interest: All authors have completed the ICMJE uniform disclosure form (available at https://dx.doi. org/10.21037/apm-21-3146). The authors have no conflicts of interest to declare.

Ethical Statement: The authors are accountable for all aspects of the work in ensuring that questions related to the accuracy or integrity of any part of the work are appropriately investigated and resolved. The study was conducted in accordance with the Declaration of Helsinki (as revised in 2013). The study was approved by the Ethics Committee of Tianjin Medical University General Hospital (approval No. 201171). Consents were provided by the participants.

Open Access Statement: This is an Open Access article distributed in accordance with the Creative Commons Attribution-NonCommercial-NoDerivs 4.0 International License (CC BY-NC-ND 4.0), which permits the noncommercial replication and distribution of the article with the strict proviso that no changes or edits are made and the original work is properly cited (including links to both the 
formal publication through the relevant DOI and the license). See: https://creativecommons.org/licenses/by-nc-nd/4.0/.

\section{References}

1. Torre LA, Bray F, Siegel RL, et al. Global cancer statistics, 2012. CA Cancer J Clin 2015;65:87-108.

2. Kamer I, Steuerman Y, Daniel-Meshulam I, et al. Predicting brain metastasis in early stage non-small cell lung cancer patients by gene expression profiling. Transl Lung Cancer Res 2020;9:682-92.

3. Waqar SN, Samson PP, Robinson CG, et al. Non-smallcell Lung Cancer With Brain Metastasis at Presentation. Clin Lung Cancer 2018;19:e373-9.

4. Khalifa J, Amini A, Popat S, et al. Brain Metastases from NSCLC: Radiation Therapy in the Era of Targeted Therapies. J Thorac Oncol 2016;11:1627-43.

5. Walker MS, Wong W, Ravelo A, et al. Effect of Brain Metastasis on Patient-Reported Outcomes in Advanced NSCLC Treated in Real-World Community Oncology Settings. Clin Lung Cancer 2018;19:139-47.

6. Qiao M, Zhao C, Liu Q, et al. Impact of ALK variants on brain metastasis and treatment response in advanced NSCLC patients with oncogenic ALK fusion. Transl Lung Cancer Res 2020;9:1452-63.

7. Ali A, Goffin JR, Arnold A, et al. Survival of patients with non-small-cell lung cancer after a diagnosis of brain metastases. Curr Oncol 2013;20:e300-6.

8. Franchino F, Rudà R, Soffietti R. Mechanisms and Therapy for Cancer Metastasis to the Brain. Front Oncol 2018;8:161.

9. Venur VA, Ahluwalia MS. Novel therapeutic agents in the management of brain metastases. Curr Opin Oncol 2017;29:395-9.

10. Malapelle U, Rolfo C. Targeted therapy and non-small cell lung cancer: a new era! Transl Cancer Res 2019;8:S1-2.

11. Ke EE, Zhou Q, Wu YL. Emerging paradigms in targeted treatments for Asian patients with NSCLC. Expert Opin Pharmacother 2015;16:1167-76.

12. Shin DY, Na II, Kim CH, et al. EGFR mutation and brain metastasis in pulmonary adenocarcinomas. J Thorac Oncol 2014;9:195-9.

13. Takamori S, Toyokawa G, Shimokawa M, et al. Radiological Features of Brain Metastases from Non-small Cell Lung Cancer Harboring EGFR Mutation. Anticancer Res 2018;38:3731-4.

14. Tang N, Guo J, Zhang Q, et al. Greater efficacy of chemotherapy plus bevacizumab compared to chemo- and targeted therapy alone on non-small cell lung cancer patients with brain metastasis. Oncotarget 2016;7:3635-44.

15. Miller JA, Kotecha R, Ahluwalia MS, et al. The impact of tumor biology on survival and response to radiation therapy among patients with non-small cell lung cancer brain metastases. Pract Radiat Oncol 2017;7:e263-73.

16. Peters S, Bexelius C, Munk V, et al. The impact of brain metastasis on quality of life, resource utilization and survival in patients with non-small-cell lung cancer. Cancer Treat Rev 2016;45:139-62.

17. Ulahannan D, Khalifa J, Faivre-Finn C, et al. Emerging treatment paradigms for brain metastasis in non-smallcell lung cancer: an overview of the current landscape and challenges ahead. Ann Oncol 2017;28:2923-31.

18. American Joint Committee on Cancer, AJCC Cancer Staging Manual. 8th edition. New York: Springer, 2017.

19. Billing PS, Miller DL, Allen MS, et al. Surgical treatment of primary lung cancer with synchronous brain metastases. J Thorac Cardiovasc Surg 2001;122:548-53.

20. Eisenhauer EA, Therasse P, Bogaerts J, et al. New response evaluation criteria in solid tumours: revised RECIST guideline (version 1.1). Eur J Cancer 2009;45:228-47.

21. Gao HX, Huang SG, Du JF, et al. Comparison of Prognostic Indices in NSCLC Patients with Brain Metastases after Radiosurgery. Int J Biol Sci 2018;14:2065-72.

22. Spence MM, Hui RL, Chang JT, et al. Treatment Patterns and Overall Survival Associated with First-Line Systemic Therapy for Patients with Advanced Non-Small Cell Lung Cancer. J Manag Care Spec Pharm 2017;23:195-205.

23. Tan PS, Bilger M, de Lima Lopes G, et al. Meta-analysis of first-line therapies with maintenance regimens for advanced non-small-cell lung cancer (NSCLC) in molecularly and clinically selected populations. Cancer Med 2017;6:1847-60.

24. David EA, Clark JM, Cooke DT, et al. The Role of Thoracic Surgery in the Therapeutic Management of Metastatic Non-Small Cell Lung Cancer. J Thorac Oncol 2017;12:1636-45.

25. Cai Y, Wang JY, Liu H. Clinical observation of whole brain radiotherapy concomitant with targeted therapy for brain metastasis in non-small cell lung cancer patients with chemotherapy failure. Asian Pac J Cancer Prev 2013;14:5699-703.

26. Jiang T, Min W, Li Y, et al. Radiotherapy plus EGFR TKIs in non-small cell lung cancer patients with brain metastases: an update meta-analysis. Cancer Med 2016;5:1055-65. 
27. Liu S, Qiu B, Chen L, et al. Radiotherapy for asymptomatic brain metastasis in epidermal growth factor receptor mutant non-small cell lung cancer without prior tyrosine kinase inhibitors treatment: a retrospective clinical study. Radiat Oncol 2015;10:118.

28. Ryoo JJ, Batech M, Zheng C, et al. Radiotherapy for brain metastases near the end of life in an integrated health care system. Ann Palliat Med 2017;6:S28-38.

29. Bugge A, Kongerud J, Brunborg C, et al. Gender-specific survival after surgical resection for early stage non-small cell lung cancer. Acta Oncol 2017;56:448-54.

30. Yoshida Y, Murayama T, Sato Y, et al. Gender Differences in Long-Term Survival after Surgery for Non-Small Cell Lung Cancer. Thorac Cardiovasc Surg 2016;64:507-14.

31. Sandler BJ, Wang Z, Hancock JG, et al. Gender, Age, and Comorbidity Status Predict Improved Survival with Adjuvant Chemotherapy Following Lobectomy for Nonsmall Cell Lung Cancers Larger than $4 \mathrm{~cm}$. Ann Surg Oncol 2016;23:638-45.

32. Ji X, Zhuang Y, Yin X, et al. Survival time following resection of intracranial metastases from NSCLCdevelopment and validation of a novel nomogram. BMC Cancer 2017;17:774.

33. Fleckenstein J, Petroff A, Schäfers HJ, et al. Longterm outcomes in radically treated synchronous vs. metachronous oligometastatic non-small-cell lung cancer.
BMC Cancer 2016;16:348.

34. Firat S, Bousamra M, Gore E, et al. Comorbidity and KPS are independent prognostic factors in stage I nonsmall-cell lung cancer. Int J Radiat Oncol Biol Phys 2002;52:1047-57.

35. Wang BX, Ou W, Mao XY, et al. Impacts of EGFR mutation and EGFR-TKIs on incidence of brain metastases in advanced non-squamous NSCLC. Clin Neurol Neurosurg 2017;160:96-100.

36. Han B, Tjulandin S, Hagiwara K, et al. EGFR mutation prevalence in Asia-Pacific and Russian patients with advanced NSCLC of adenocarcinoma and nonadenocarcinoma histology: The IGNITE study. Lung Cancer 2017;113:37-44.

37. Zhang YL, Yuan JQ, Wang KF, et al. The prevalence of EGFR mutation in patients with non-small cell lung cancer: a systematic review and meta-analysis. Oncotarget 2016;7:78985-93.

38. Sung S, Lee SW, Kwak YK, et al. Intracranial control and survival outcome of tyrosine kinase inhibitor (TKI) alone versus TKI plus radiotherapy for brain metastasis of epidermal growth factor receptor-mutant non-small cell lung cancer. J Neurooncol 2018;139:205-13.

(English Language Editor: J. Jones)
Cite this article as: Xue J, Liu M, Zhao H, Li X, Qin Q, Ren Y, Zhong D. The role of brain metastases in patients with pulmonary adenocarcinoma: a retrospective study. Ann Palliat Med 2021;10(12):12262-12272. doi: 10.21037/apm-21-3146 